\title{
Recombinant tegumental protein Shistosoma japonicum very lowdensity lipoprotein binding protein as a vaccine candidate against Schistosoma japonicum
}

\author{
Xiao-Xian Gan, Li-Ying Shen, Yue Wang, Jian-Zu Ding, Hui-Ying Shen, Xiao-Peng Zeng, \\ Donald P M cManus*, Paul J Brindley** $/^{+}$, Jinjiang Fan $/ * * * /+$
}

Institute of Parasitic Diseases, Zhejiang Academy of Medical Sciences, Hangzhou 310013, PR China *Molecular Parasitology Unit, Australian Centre for International and Tropical Health and Nutrition, The Queensland Institute of Medical Research and The University of Queensland, Brisbane, Queensland, Australia **Department of Tropical Medicine, Tulane University

Health Sciences Center, New Orleans, Louisiana, US ***Guy-Bernier Research Center, Maisonneuve-Rosemont Hospital, Montreal, Canada

A polyhistidine-tagged recombinant tegumental protein Schistosoma japonicum very lowdensity lipoprotein binding protein (SVLBP) from adult Schistosoma japonicum was expressed in Escherichia coli. The affinity purified $r S V L B P$ was used to vaccinate mice. The worm numbers and egg deposition recovered from the livers and veins of the immunized mice were $33.5 \%$ and $47.6 \%$ less than that from control mice, respectively $(p<0.05)$. There was also a marked increase in the antibody response in vaccinated mice: the titer of IgGI and IgG2a, IgG2b in the vaccinated group was significantly higher than that in the controls ( >1:6,400 in total IgG). In a comparison of the reactivity of sera from healthy individuals and patients with rSVLBP, recognition patterns against this parasite tegumental antigen varied among different groups of the individuals. Notably, the average titres of anti-rSVLBP antibody in sera from faecal egg-negative individuals was significantly higher than that in sera from the faecal egg-positives, which may be reflect $S V L B P$-specific protection. These results suggested that the parasite tegumental protein SVLBP was a promising candidate for further investigation as a vaccine antigen for use against Asian schistosomiasis.

Key words: Schistosoma japonicum - Schistosoma japonicum very lowdensity lipoprotein binding protein - vaccine

Schistosomiasis, also known as bilharzia, is the major health risk in tropical and subtropical countries around the world, which affects more than 200 million individuals in 74 countries and remains among the most debilitating parasitic diseases of humanity (Engels et al. 2002, Ross et al. 2002, Chitsulo et al. 2004). Praziquantel, the current drug of choice for all forms of human schistosomiasis, is safe and effective. However, efficient drug delivery requires a substantial infrastructure to regularly cover all parts of an endemic area and, moreover, in endemic regions people often are re-infected soon after drug cure. In addition, there are continued concerns about the emergence of drug resistance to praziquantel (Cioli et al. 2004). All of these reasons have accelerated research aimed at development of a vaccine for schistosomiasis. Development of a vaccine against the disease remains a worthwhile and desirable goal in view of controlling the transmission of schistosomiasis (Chitsulo et al. 2004, McManus \& Bartley 2004, McManus 2005).

Financial support: UNDP/World Bank/WHO Special Program for Research and Training in Tropical Diseases to JF (ID 981115) and the Science \& Technology Bureau of Zhejiang Province, PR China to X-X.G. (001106129, 001103257). PJB is a recipient of a Burroughs Wellcome Fund Scholar Award in Molecular Parasitology.

${ }^{+}$Corresponding authors: paul.brindley@tulane.edu and jinjiang.fan@gmail.com

Received 30 May 2005

Accepted 27 December 2005
Although numerous, promising vaccine candidates against S. japonicum have been studied so far, none of them have been proven to be as effective as irradiated cercariae, the gold standard in terms of an experimental anti-schistosome vaccine (reviewed in Bergquist et al. 2002, Pearce 2003, McManus \& Bartley 2004). Recent studies aimed at sequencing the genome of $S$. japonicum have provided new sources for the development of new target antigens (Fan et al. 1998, Hu et al. 2003). In our previous report, an expressed sequence tag (EST) of S. japonicum encoding a $S$. japonicum very low-density lipoprotein binding protein (SVLBP) arose as a interesting candidate for further study its biological functions and role in the host-schistosome relationship (Fan et al. 2003). SVLBP was reported to be membrane-associated, and located in the tegument and sub-tegument of adult male schistosomes (Fan et al. 2003). Given that the SVLBP may play an essential role in lipid acquisition by the parasite, and/or in signal transduction pathways, we considered that it was worthy of further investigation for development as a novel anti-schistosomal intervention. Here we report the expression and purification of the recombinant SVLBP for investigation of SVLBP as an experimental vaccine, vaccination of mice with affinity purified, recombinant SVLBP, the IgG subclass antibody responses in the vaccinated mice, and anti-rSVLBP antibody responses in sera of humans naturally infected with S. japonicum.

\section{MATERIALS AND METHODS}

Parasites, experimental animals, and patients - A Chinese mainland isolate of S. japonicum from the Anhui 
Province was maintained in the laboratory by infecting Oncomelania hupensis hupensis snails and Netherlands rabbits. Active cercariae were freshly shed from the infected snails under light irradiation and used for infection of rabbits or mice. Female mice C57BL/6 were purchased from the Experimental Animal Center, Zhejiang Province, PR China. In addition, 253 sera samples were collected from human subjects residing in S. japonicum endemic regions of Jiangxi Province, PR China. Stool samples of these volunteers were examined for $S$. japonicum eggs using the modified Kato-Katz thick smear method (Katz et al. 1972). The faecal egg positive individuals were offered treatment for schistosomiasis with praziquantel. Furthermore, human serum samples were also obtained from 105 other individuals residing in non-schistosome endemic regions (Hangzhou City, Zhejiang Province) in PR China.

Recombinant SVLBP - A cDNA encoding SVLBP was cloned into pQE30 (Qiagen) and the expression of the recombinant protein was induced with a final concentration of $1 \mathrm{mM}$ isopropyl-beta-D-thiogalactopyranoside (IPTG) at $37^{\circ} \mathrm{C}$ for $4 \mathrm{~h}$ with shaking after transformation into E. coli (Fan et al. 2003). The recombinant SVLBP was purified on Ni-NTA Superflow resin by affinity chromatography under native conditions, using a linear imidazole gradient (10-250 mM). Eluted fractions containing the expressed recombinant protein were pooled and concentrated in PBS buffer $\left(20 \mathrm{mM} \mathrm{NaH}_{2} \mathrm{PO}_{4}, 30 \mathrm{mM} \mathrm{NaCl}, \mathrm{pH}\right.$ 7.5). The presence of the recombinant protein was confirmed by SDS-PAGE analysis. Protein concentration was determined by Lowry's method (Markwell et al. 1978).

Immunization of mice with the recombinant SVLBP Female C57BL/6 mice, 18-22 g in weight and 6-8 weeks old were randomly divided into two groups. Thirteen mice in the experimental group were each injected subcutaneously (sc) with $45 \mu 1$ phosphate buffered saline containing $45 \mu \mathrm{g}$ purified recombinant SVLBP plus complete Freund's adjuvant at multiple sites of the abdominal area, at weeks $0,2,4$, respectively. Twelve mice in the control group received the same volume of PBS plus adjuvant as mice in the immunization group. Serum samples were obtained before vaccination and at the time of necropsy, respectively.

Cercarial challenge, worm recovery and egg count Mice of both groups were challenged percutaneously with $35 \pm 1$ cercariae by cover slip method (Ruppel et al. 1990) two weeks after the final vaccine or control injection. Mice were euthanized 45 days after the challenge infection by overdose of heparinized sodium pentobarbitone (Sigma) administered by intraperitoneal injection. Immediately thereafter, the portal system blood vessels perfused in order to recover the worms from the livers and veins. Subsequently, the liver were each weighed and minced and digested in $10 \mathrm{ml}$ of $5 \% \mathrm{KOH}$ at $37^{\circ} \mathrm{C}$ for $24 \mathrm{~h}$ after which schistosome eggs released from the infected livers were counted in $100 \mu \mathrm{l}$ aliquots. Five aliqouts were counted for per liver samples (Liu et al. 1995) aided by light microscopic examination.

Enzyme-linked immunosorbent assay - Serum antiSVLBP antibody responses of immunized mice were measured by ELISA. The plates were coated with $2 \mu \mathrm{g} /$ well of recombinant SVLBP in carbonate/bicarbonate buffer $(\mathrm{pH}$ 9.6). After blocking with $3 \%$ (w/v) BSA in PBS/0.05\% (v/ v) Tween-20 (PBS-T20), mouse sera were diluted $1 / 100$ in PBS-T20 and added $(100 \mu \mathrm{l} /$ well $)$, then incubated at $37^{\circ} \mathrm{C}$ for $1 \mathrm{~h} .100 \mu \mathrm{l} /$ well of horseradish peroxidase (HRP)-labeled anti-mouse IgG, IgG1, IgG2a, IgG2b, IgG3 (Caltag Laboratories, Inc. US) diluted 1/2000 was added and incubated at $37^{\circ} \mathrm{C}$ for $1 \mathrm{~h}$. One $\mathrm{mg} / \mathrm{ml}$ TMB $\left(3,3^{\prime}, 5,5^{\prime}\right.$ tetramethylbenzidene) was used as the substrate, and the absorbance was read at $450 \mathrm{~nm}$ in a spectrophotomer 15 min after incubation.

Statistical analysis - The non-parametric $t$-test and/ or Mann-Whitney U test were used to determine the significance of differences in mean worms and egg counts from the immunized groups and the adjuvant control, and to determine the human antibody responses to rSVLBP. All results including the ELISA optical density (OD) values are presented as mean \pm S.D. A $p$ value of $\leq 0.05$ was considered statistically significant.

\section{RESULTS}

Expression of the recombinant SVLBP in E. coli and its purification by affinity chromatography - Expression of the recombinant SVLBP was achieved by introduction of IPTG to the culture, resulting in that a $22 \mathrm{kDa}$ protein was induced (Fig. 1), which was in accordance with the previous description (Fan et al. 2003). The 22 kilodaltons was approximately the size expected of a full-length recombinant fusion protein, i.e. about $20 \mathrm{kDa}$ for SVLBP and $2 \mathrm{kDa}$ for the $\mathrm{NH}_{2}$-terminal fusion peptide incorporating the six-histidine tag. A single-step solubilization/purification on Ni-NTA resin under non-denaturing conditions was used to purify the recombinant SVLBP in native form. This chromatographic procedure was able to enrich the SVLBP almost to homogeneity (Fig. 1).

Protective efficacy of $r$ SVLBP against $S$. japonicum Two groups of mice were used as experiment and control, respectively. Group 1 was immunized with $\operatorname{rSVLBP}(\mathrm{n}=$ 13 ), and group 2 was injected with adjuvant as control (n $=12$ ). Worm numbers and liver egg counts for immunized and control groups are presented in the Table. The mean number $(X \pm$ S.D. ) of worms recovered from mice vaccinated with $\mathrm{rSVLBP}(15.5 \pm 6.1)(\mathrm{n}=13)$ was significantly reduced by $33.5 \%(p<0.05)$ compared with the mean worm numbers from control mice $(23.3 \pm 6.8)(n=12)$. There was also a significant $47.6 \%$ decrease $(p<0.05)$ in mean number of eggs in livers from vaccinated mice $\left(9.7 \pm 2.7 \times 10^{3}\right.$; $\mathrm{n}=13$ ) compared with the mean number of eggs in livers from control mice $\left(18.5 \pm 4.2 \times 10^{3} ; \mathrm{n}=12\right)$.

Increase of the specific anti-rSVLBP antibodies after parasite challenge - Anti-rSVLBP IgG antibody levels (mean absorbance values at $450 \mathrm{~nm} \pm$ S.D.) were measured in sera of vaccinated and control mice (Fig. 2). Antibody responses to mice immunized with $\operatorname{rSVLBP}(2.167 \pm 0.34)$ were significantly higher than antibody levels in sera of mice injected with adjuvant only $(0.039 \pm 0.022 ; p<0.01)$. Distinct antibody titres as high as 1:6400 were obtained from immunized group for the total $\mathrm{IgG}$, indicating that rSVLBP may be an ideal immunogen candidate in that it elicits strong antibody responses. The profile of specific $\operatorname{IgG}$ subclasses after challenge was dominated by $\operatorname{IgG} 1$, 


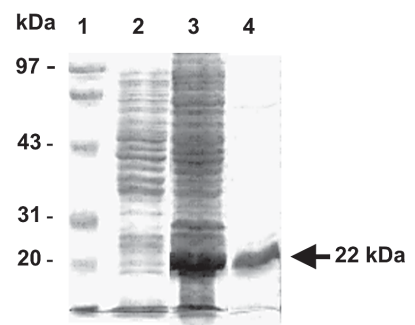

Fig. 1: SDS-PAGE and Coomassie stained gel analysis of the expression of recombinant SVLBP in E. coli and affinity purification. Lanes - 1: molecular size standards (kDa); 2: uninduced bacterial cell lysates; 3 : bacterial cell lysates after induced $3 \mathrm{~h}$ by adding IPTG to $1 \mathrm{mM}$; 4: purified recombinant SVLBP (rSV LBP). The arrow indicates the position of rSVLBP.

$\operatorname{IgG} 2 \mathrm{a}$, and $\operatorname{IgG} 2 \mathrm{~b}$, but not by $\operatorname{IgG} 3$. The $\operatorname{IgG} 1 / \operatorname{IgG} 2$ ratios in the vaccinated mice were less than 0.62 . There was no significant detectable specific antibody response between the control group and before immunization (Fig. 2).

Recognition of the rSVLBP by human sera from schistosomiasis endemic and non-endemic regions - Antigenicity of rSVLBP was measured in 253 sera from individuals from endemic regions where schistosomiasis japonica is rampant and in sera from 105 uninfected volunteers from non-endemic regions. Human sera from the individuals in endemic regions with positive - or negative - faecal examinations exhibited higher responses than those from healthy individuals in non-endemic regions. It is noteworthy that antibody responses to rSVLBP were significantly greater in the individuals with faecal examination negative versus the patients with faecal examination positive (Mann-Whitney U test, $p<0.006$ ) (Fig. 3). Together, these data confirm that rSVLBP displays strong immune responses during the course of natural infection, and suggests a protective effect in sero-positive, faecal negative individuals.

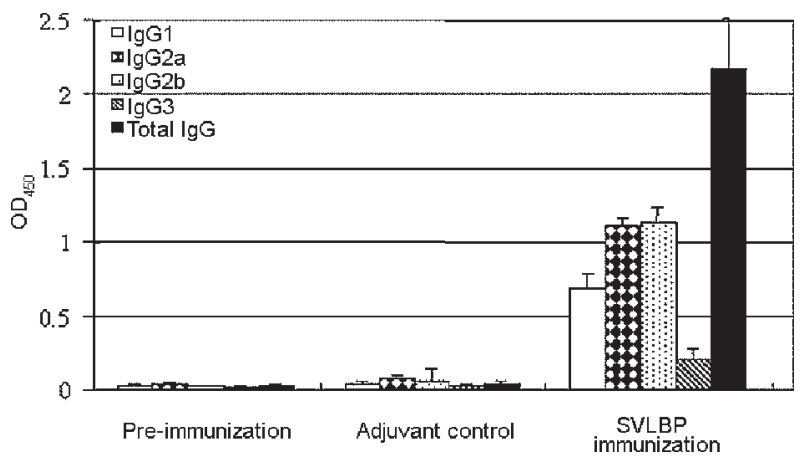

Fig. 2: levels of serum IgG isotype antibody responses in C57BL/6 mice detected by ELISA to rSVLBP compared for rSVLBP immunization (group 1), adjuvant control (group 2), and before immunization. Bars represent the mean $\pm \mathrm{SE}$.

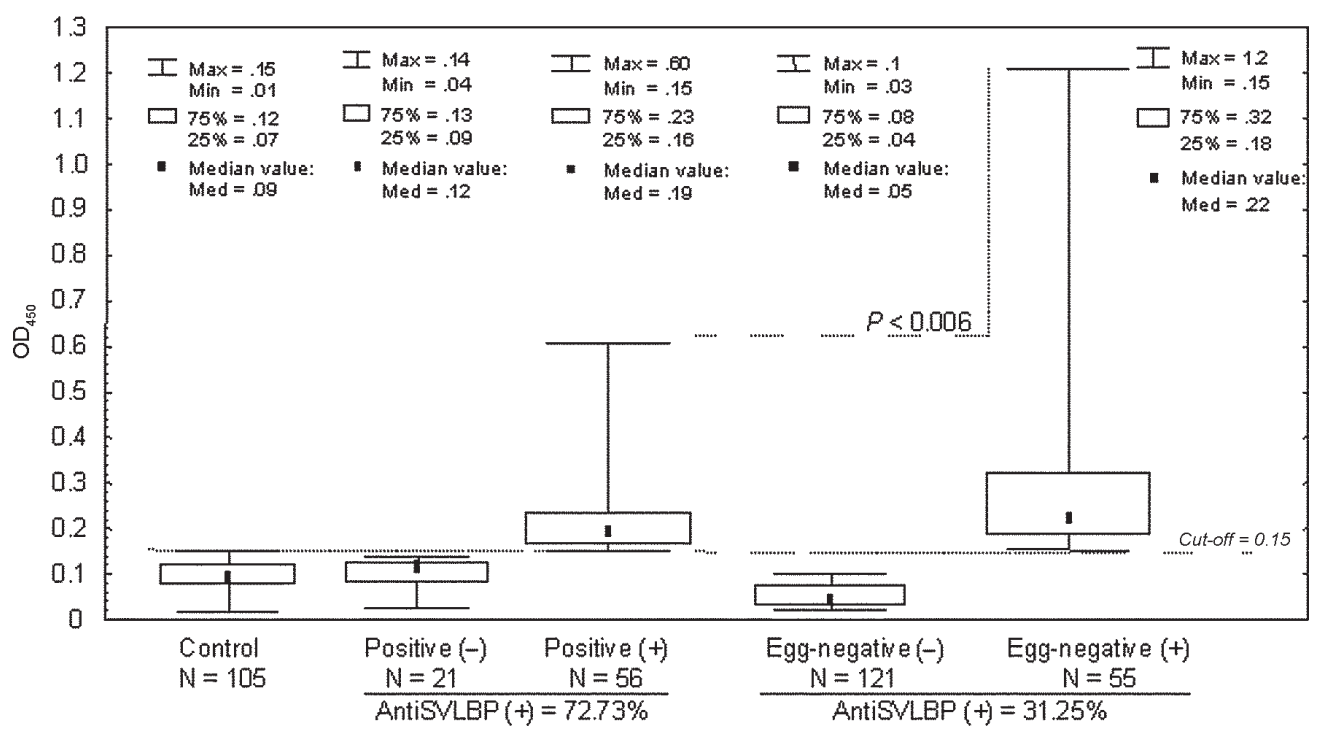

Fig. 3: reactivity of 358 sera from endemic regions and non-endemic (control) with rSVLBP antigens using ELISA test for intestinal schistosomiasis. The box and whisker plot (type: median/quart/range) shows the median and range values of serum anti-rSVLBP levels. The median response is represented by a black filled box within a rectangular box that represents the 25th to 75 th percentile range. Whiskers represent the full range of our actual data. Although the $\%$ of the positive reaction to rSVLBP in the patients with a negative faecal egg counts was significantly lower compared to the patients with positive faecal examination, there was a remarkable elevated level of antirSVLBP antibody in the faecal egg counts-negative group (Mann-Whitney $\mathrm{U}$ test, $p<0.006$ ).

TABLE

Worm burden and eggs per gram of liver in mice vaccinated with rSVLBP after challenge infection of Schistosoma japonicum

\begin{tabular}{|c|c|c|c|c|c|c|c|}
\hline \multirow[t]{2}{*}{ Group } & \multirow[t]{2}{*}{ No. of mice } & \multicolumn{3}{|c|}{ Worm burden } & \multicolumn{3}{|c|}{ Eggs per gram of liver tissue } \\
\hline & & $\mathrm{X} \pm \mathrm{SD}$ & Reduction & $p$ & $\mathrm{X} \pm \mathrm{SD}$ & Reduction & $p$ \\
\hline Recombinant SVLBP & 13 & $15.5 \pm 6.1$ & $33.5 \%$ & $<0.05$ & $9726 \pm 2722$ & $47.6 \%$ & $<0.05$ \\
\hline Adjuvant control & 12 & $23.3 \pm 6.8$ & & & $18543 \pm 4242$ & & \\
\hline
\end{tabular}




\section{DISCUSSION}

The schistosomule tegument is an enigmatic structure - a double bilayer - that is involved with the transition from free-living to the parasitic life style in the mammalian host, in immunological masking, and in escape from immune defenses. In addition, it plays a vital role in acquisition of host derived nutrients, including carbohydrates, amino acids, and lipids (Abath \& Werkhauser 1996, Jones et al. 2004). Along with other key molecules, tegument associated antigens of schistosomes have been investigated as targets of protective immune responses in murine hosts and for defining antibody and cytokine correlates of apparent resistance and susceptibility in human populations, a key approach to establishing the protective potential of a vaccine candidate (Ribeiro de Jesus et al. 2000, Acosta et al. 2002, 2004, Al-Sherbiny et al. 2003, McManus \& Bartley 2004, Bergquist et al. 2005). Since SVLBP appears to have VLDL binding activity and to locate prominently on the tegument and sub-tegument of adult male schistosomes, SVLBP may play a key role in lipid acquisition by S. japonicum (Fan et al. 2003). Given that anti-SVLBP antibody can block lipid binding capacity of the SVLBP, blockage of SVLBP by antibodies and other immune effectors may result in starving the parasite of essential lipid nutrients from the host and may also disrupt lipid molecule-associated immune evasion processes (Fan et al. 2003). Here we have shown that, when delivered as a vaccine, rSVLBP can induce a significant level of protection evidenced as reduced worm burden and liver egg counts in the vaccinated mice. The level of protection achieved was comparable to that reported with a number of other experimental vaccine preparations, including with many S. mansoni antigens tested in experimentally infected mice (reviewed in Pearce 2003, McManus 2005).

Profiling of antibody levels of anti-rSVLBP antibodies revealed that $\mathrm{IgG} 2 \mathrm{a}$ and $\mathrm{IgG} 2 \mathrm{~b}$ antibody subclasses predominated in the serum of the vaccinated mice. Gamma interferon (IFN-gamma) and interleukin 4 ( $=$ B cell stimulatory factor-1, BSF-1), which are T cell-derived lymphokines, can stimulate B cells to produce $\operatorname{IgG} 2$ a and $\mathrm{IgG} 1$, respectively. Therefore, the IgG2a and IgG1 can be used as serological markers to indicate the induction of Th1/ $\mathrm{Tc} 1$ and $\mathrm{Th} 2 / \mathrm{Tc} 2$ immune responses, respectively (Snapper \& Paul 1987). In general, IgG1/IgG2 ratio of < 1.0 indicates a predominantly Th1 type of response whereas IgG1/ $\mathrm{IgG} 2$ ratio of $>1.0$ indicates a Th2 phenotype. The increase in both $\operatorname{IgG} 2 \mathrm{a}$ and $\operatorname{IgG} 2 \mathrm{~b}$ seen in the present study clearly suggests that a predominantly $\mathrm{Th} 1 / \mathrm{Tc} 1$ type of antibody response contributed to the protective response induced by vaccination with rSVLBP. Previous reports on vaccination against $S$. mansoni challenge in mice have shown that only immunized mice develop higher levels of IgG2a (and lower levels of IgG1) to larval and adult worm antigens (Mountford et al. 1994). In addition, using DNA encoding $S$. mansoni $28 \mathrm{kDa}$ glutathione $S$-transferase to immunize rats leads to a long-lasting predominance of IgG2a and IgG2b (Dupre et al. 1997), as does the p80 subunit of S. mansoni calpain (Siddiqui et al. 2003). DNA vaccination and co-delivery of antigens with IL-12 result in amplified IgG2a synthesis and down-regulation of synthesis of IgG1 (Fonseca et al. 2004, Siddiqui et al. 2003). Interestingly, our study demonstrated that vaccination with rSVLBP induced a predominantly a Th1-type of immune response in mice. This suggests that the vaccine effect was likely to have been mediated by an antibodydependent, cell-mediated cytotoxicity (ADCC) response involving parasite killing the parasite by monocytes, eosinophils and/or platelets (Capron et al. 1983, Horta \& Ramalho-Pinto 1984, 1987).

This work provides the first evidence that the newly discovered S. japonicum-specific molecule, SVLBP, has potential as a vaccine candidate. Further, the immune recognition of rSVLBP in human sera from individuals from schistosomiasis japonica endemic regions confirms that the rSVLBP can elicit an immune response in natural human populations in endemic regions, which is a requirement for a potential vaccine candidate. In future studies it would be instructive to clarify the immuno-modulatory mechanisms responsible for its vaccine action along the lines reported by Capron and coworkers with recombinant $28 \mathrm{kDa}$ glutathione-S-transferase of Schistosoma haematobium (rShGST28), which represents the first schistosome vaccine tested in humans. Capron et al. (2005) noted that, in initial trials, rShGST28 produced in yeast and delivered in alum is safe and that it can engender significant antischistosomal effects. Given that $S$. japonicum infection is a true zoonosis, unlike the other species of schistosomes of public health consequence, it has been suggested that if a transmission blocking veterinary vaccine developed for bovines can be put into practice in combination with other control strategies such as human chemotherapy, elimination of S. japonicum from China may be achievable (McManus 2005). Furthermore, it has recently become clear that vaccine-linked chemotherapy associated with the artemisinin derivative artemether offers the potential for an integrated - chemotherapy and vaccination - approach to schistosomiasis control (Bergquist et al. 2005). On both these fronts also, SVLBP appears to be worthy of further investigation as a transmission blocking, and chemotherapy-linked, vaccine.

\section{REFERENCES}

Abath FG, Werkhauser RC 1996. The tegument of Schistosoma mansoni: functional and immunological features. Parasite Immunol 18: 15-20.

Acosta LP, McManus DP, Aligui GD, Olveda RM, Tiu WU 2004. Antigen-specific antibody isotype patterns to Schistosoma japonicum recombinant and native antigens in a defined population in Leyte, the Philippines. Am J Trop Med Hyg 70: 549-555.

Acosta LP, Waine G, Aligui GD, Tiu WU, Olveda RM, McManus DP 2002. Immune correlate study on human Schistosoma japonicum in a well-defined population in Leyte, Philippines: II. Cellular immune responses to $S$. japonicum recombinant and native antigens. Acta Trop 84 : 137-149.

Al-Sherbiny M, Osman A, Barakat R, El Morshedy H, Bergquist $\mathrm{R}$, Olds R 2003. In vitro cellular and humoral responses to Schistosoma mansoni vaccine candidate antigens. Acta Trop 88: 117-130. 
Bergquist NR, Leonardo LR, Mitchell GF 2005. Vaccine-linked chemotherapy: can schistosomiasis control benefit from an integrated approach? Trends Parasitol 21: 112-117.

Bergquist R, Al-Sherbiny M, Barakat R, Olds R 2002. Blueprint for schistosomiasis vaccine development. Acta Trop 82: 183-192.

Chitsulo L, Loverde P, Engels D 2004. Schistosomiasis. Nat Rev Microbiol 2: 12-13.

Capron M, Capron A, Abdel-Hafez SK, Bazin H, Joseph M, Phillips SM 1983. Immunologic response of athymic rats to Schistosoma mansoni infection. II. Antibody-dependent mechanisms of resistance. J Immunol 131: 1475-1480.

Capron A, Riveau G, Capron M, Trottein F 2005. Related articles, schistosomes: the road from host-parasite interactions to vaccines in clinical trials. Trends Parasitol 21: 143149.

Cioli D, Botros SS, Wheatcroft-Francklow K, Mbaye A, Southgate V, Tchuente LA, Pica-Mattoccia L, Troiani AR, El-Din SH, Sabra AN, Albin J, Engels D, Doenhoff MJ 2004. Determination of ED50 values for praziquantel in praziquantel-resistant and -susceptible Schistosoma mansoni isolates. Int J Parasitol 34: 979-87.

Dupre L, Poulain-Godefroy O, Ban E, Ivanoff N, Mekranfar M, Schacht AM, Capron A, Riveau G 1997. Intradermal immunization of rats with plasmid DNA encoding Schistosoma mansoni $28 \mathrm{kDa}$ glutathione S-transferase. Parasite Immunol 19: 505-513.

Engels D, Chitsulo L, Montresor A, Savioli L 2002. The global epidemiological situation of schistosomiasis and new approaches to control and research. Acta Trop 82: 139-146.

Fan J, Gan X, Yang W, Shen L, McManus DP, Brindley PJ 2003. A Schistosoma japonicum very low-density lipoprotein-binding protein. Int J Biochem Cell Biol 35: 1436-1451.

Fan J, Minchella DJ, Day SR, McManus DP, Tiu WU, Brindley PJ 1998. Generation, identification, and evaluation of expressed sequence tags from different developmental stages of the Asian blood fluke Schistosoma japonicum. Biochem Biophys Res Commun 252: 348-356.

Fonseca CT, Brito CF, Alves JB, Oliveira SC 2004. IL-12 enhances protective immunity in mice engendered by immunization with recombinant $14 \mathrm{kDa}$ Schistosoma mansoni fatty acid-binding protein through an IFN-gamma and TNFalpha dependent pathway. Vaccine 22: 503-510.

Horta MF, Ramalho-Pinto FJ 1984. Subclasses of rat IgG active in the killing of schistosomula of Schistosoma mansoni in vitro and in vivo. J Immunol 133: 3326-3332.

Horta MF, Ramalho-Pinto FJ 1987. Levels of lethal antibody during the course of infection with Schistosoma mansoni in rats and mice. Mem Inst Oswaldo Cruz 82 (Suppl. 4): 283284.

Hu W, Yan Q, Shen DK, Liu F, Zhu ZD, Song HD, Xu XR,
Wang ZJ, Rong YP, Zeng LC, Wu J, Zhang X, Wang JJ, Xu XN, Wang SY, Fu G, Zhang XL, Wang ZQ, Brindley PJ, McManus DP, Xue CL, Feng Z, Chen Z, Han ZG 2003. Evolutionary and biomedical implications of a Schistosoma japonicum complementary DNA resource. Nat Genet 35: 139-147.

Jones MK, Gobert GN, Zhang L, Sunderland P, McManus DP 2004. The cytoskeleton and motor proteins of human schistosomes and their roles in surface maintenance and hostparasite interactions. Bioessays 26: 752-765.

Katz N, Chaves A, Pellegrino J 1972. A simple device for quantitative stool thick-smear technique in schistosomiasis mansoni. Rev Inst Med Trop São Paulo 14: 397-400.

Liu SX, Song GC, Xu YX, Yang W, McManus DP 1995. Immunization of mice with recombinant Sjc26GST induces a pronounced antifecundity effect after experimental infection with Chinese Schistosoma japonicum. Vaccine 13: 603-607.

Markwell MA, Haas SM, Bieber LL, Tolbert NE 1978. A modification of the Lowry procedure to simplify protein determination in membrane and lipoprotein samples. Anal Biochem 87: 206-210.

McManus DP 2005. Prospects for development of a transmission blocking vaccine against Schistosoma japonicum. Parasite Immunol 27: 297-308.

McManus DP, Bartley PB 2004. A vaccine against Asian schistosomiasis. Parasitol Int 53: 163-173.

Mountford AP, Fisher A, Wilson RA 1994. The profile of IgG1 and IgG2a antibody responses in mice exposed to Schistosoma mansoni. Parasite Immunol 16: 521-527.

Pearce EJ 2003. Progress towards a vaccine for schistosomiasis. Acta Trop 86: 309-313.

Ribeiro de Jesus A, Araujo I, Bacellar O, Magalhaes A, Pearce E, Harn D, Strand M, Carvalho EM 2000. Human immune responses to Schistosoma mansoni vaccine candidate antigens. Infect Immun 68: 2797-2803.

Ross AG, Bartley PB, Sleigh AC, Olds GR, Li Y, Williams GM, McManus DP 2002. Schistosomiasis. N Engl J Med 346: 1212-1220.

Ruppel A, Shi YE, Moloney NA 1990. Schistosoma mansoni and S. japonicum: comparison of levels of ultraviolet irradiation for vaccination of mice with cercariae. Parasitology 101: 23-26.

Siddiqui AA, Phillips T, Charest H, Podesta RB, Quinlin ML, Pinkston JR, Lloyd JD, Pompa J, Villalovos RM, Paz M 2003. Enhancement of Sm-p80 (large subunit of calpain) induced protective immunity against Schistosoma mansoni through co-delivery of interleukin-2 and interleukin-12 in a DNA vaccine formulation. Vaccine 21: 2882-2889.

Snapper CM, Paul WE 1987. Interferon-gamma and B cell stimulatory factor-1 reciprocally regulate Ig isotype production. Science 236: 944-947. 
